\title{
Effect of Different Window Size on Median Filter Performance with Variable Noise Densities
}

\author{
Asmaa Hameed Rasheed \\ Lecturer \\ College of Engineering, Baghdad University \\ Baghdad, Iraq
}

\begin{abstract}
A noise removal (de-noising) is one of the important problems in image processing applications. The noise added to the original image by changes the intensity of some pixels while other remain unchanged. Salt-andpepper noise is one of the impulse noises, to remove it a simplest way used by windowing the noisy image with a conventional median filter. Median filters are the most popular filters extensively applied to eliminate salt-andpepper noise. This paper evaluates the performance of median filter based on the effective median per window by using different window sizes. The experimental results show that median filter has a good performance in low noise densities and also in high noise densities when using high level of window sizes, but with higher window size a degree of blurring effect will be added to filtered noise.
\end{abstract}

The approach used is a windowing operator technique to cut the pixels of an image, and apply filtering processing to them that take different window sizes $3 * 3$ and $5 * 5$ and $7 * 7$. The results obtain for image size of $250 * 400$.

\section{Keywords}

Image filtering, median filter, gray image, salt \& pepper noise

\section{INTRODUCTION}

Digital Image Processing becomes a major field of study in the area of computer science and communication engineering. So, all systems which were operating on the traditionally analog imaging are now gradually switching to the digital systems for their ease of use, affordability and flexibility. Conversion of images from analog form to digital form causes an impulse noise to appear during image processing by a camera, scanner, and recording in most types of systems such TV stations or Satellites, and when the image is transmitted over a noisy channel. Image processing is very important and has been extensively used in widely different applications area: such as medicine, photography, remote sensing satellites, military target analysis, and manufacturing automation \& control [1]. Salt-and-pepper noise is a special case of impulse noise, where a certain percentage of individual pixels are randomly digitized into two extreme intensities, maximum and minimum. The need to remove salt-and-pepper noise is very important before subsequent image processing tasks are carried out because the contamination of image by salt-and-pepper noise is caused in great amount and the occurrence of noise can severely damage the information or data contained in the original image. Different noise filtering techniques can be useful with this noise type. Median filter is most filter used to remove the salt and pepper

\author{
Haneen Mohammed Hussein \\ Student \\ College of Engineering, Baghdad University \\ Baghdad, Iraq
}

noise it uses a technique of windowing pixels of noisy image. The pixels in the window are first sorted and the center pixel is changed to the median value of the sorted sequence. This method is the simplest of the median filtering techniques and because of its simplicity [2].

In this work the performance of median filter is evaluated based on different window sizes and study the effects of variation of window size in order to remove the salt and pepper noise from gray image.

\section{MEDIAN FILTER ALGORITHM}

The median filter is a nonlinear digital filtering technique, often used to remove salt and pepper noise [3]. Median filtering is very widely used in digital image processing because, under certain conditions, it preserves edges of the images while removing noise and it's a nonlinear local filter whose output value is the middle element of a sorted array of pixel values from the filter window. The median filter filters each pixel in the image in turn and its nearby neighbors are used to decide whether or not it is representative of its surroundings [4]. Median filter replaces the pixel value with the median of those values. That is, the values from the surrounding neighborhood are first sorted into numerical order, and then the value of the pixel in question is replaced with the middle (median) pixel value. The neighborhood is referred to as the window. The window can have various shapes centered on the target pixel, typical a square shape chosen for windows defined for 2D images. The median value of the surrounding pixels is most likely to be the value of one of the pixels in the neighborhood within the window. Thus the median filter is least likely to create new unrealistic pixel values.

An important property of the median filter is that its output is always constrained, by definition, to be the median value in the window. In a $\mathrm{k} \times \mathrm{k}$ window, if the number of masked pixels is very large, then the median computed will be an impulse and the noise will not be removed. On the other hand the center value replaced is not tested to find out if it is an impulse or not. Hence if it is not an impulse but a fine pixel of the image then it is removed unnecessarily. The median filter performs poorly when the intensity of the noise is high [5].

The median filter mask apply to calculating the brightness of pixel and determining which brightness value is the median value. This filter good for "salt and pepper" noise, which is noise that represents itself as randomly occurring black and white pixels on an image. Salt and pepper noise creeps into images in situations where quick transients, such as faulty switching, take place in an image capture device [6]. 


\section{ALGORITHM OF THE PROPOSED TECHNIQUE}

The proposed technique can be summarized into the following steps:

Input the gray image.

Add zero padding.

Determine type of the tested noise.

Determine the window size of median filter.

Process the hole data.

Re-frame and display image.

The algorithm of this work can represent in flow chart in Figure 1.

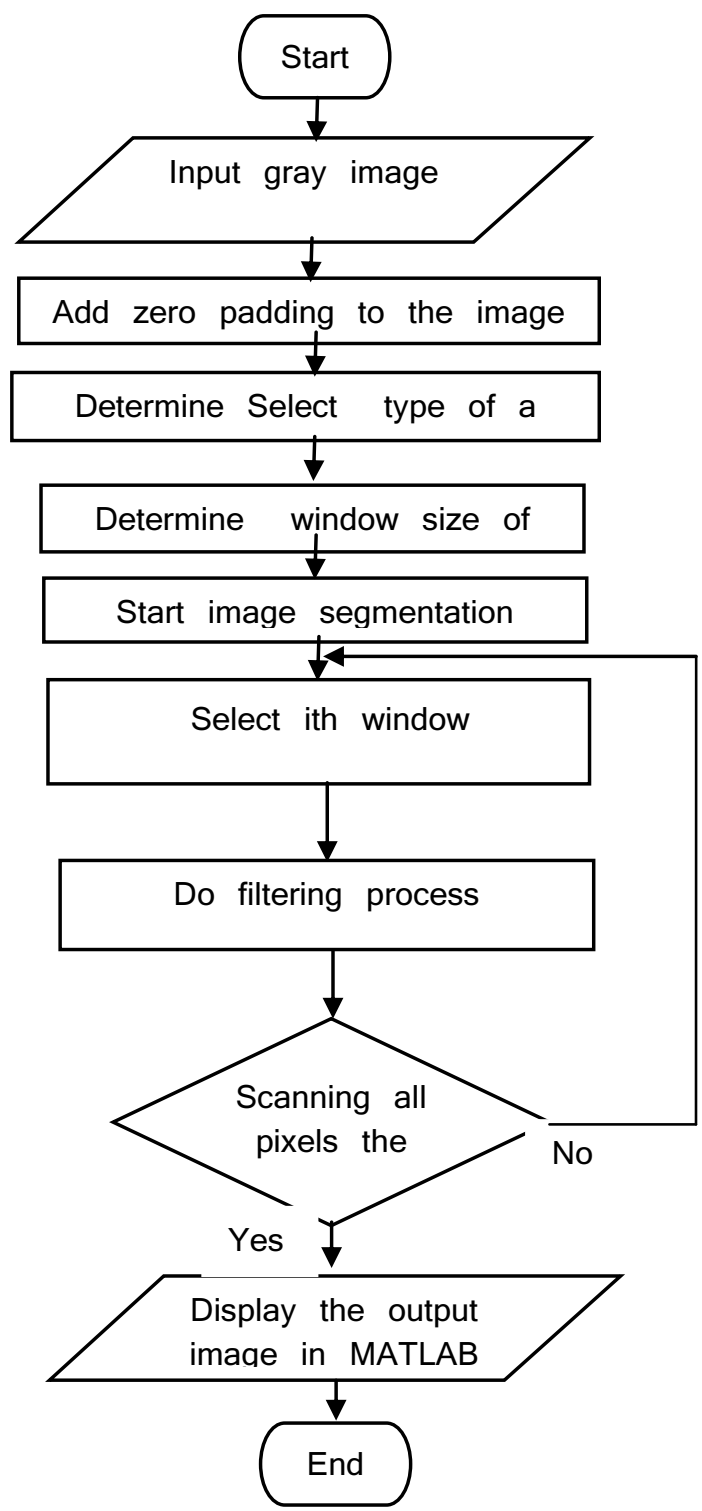

Fig 1: Flow chart of the proposed technique

\section{EVALUATION OF IMAGE QUALITY}

There are several techniques and metrics that can be measured objectively and automatically evaluated by a computer program [7]. Therefore, they can be classified[2] as:

1. Full-reference (FR) methods - FR metrics try to assess the quality of a test image by comparing it with a reference image that is assumed to have perfect quality.

2. No-reference (NR) methods - NR metrics try to assess the quality of a test image without any reference to the original one.

On the other hand, for subjective quality evaluation, the subjective scores given by human observers are used as the objective values in the analysis. The predictive linear regression models for the objective and subjective image quality evaluations, which were constructed using training images, were then used on test data wherein the calculated objective values are construed as the evaluation indices.

For objective quality evaluation, the mean square of the minimum differences of all pixels in the entire image is defined as the evaluation measure for noise:

$\mathrm{n}=\frac{1}{M} \sum_{1}^{M}[\operatorname{dmin}(i)]^{2}$

\section{EXPEREMENTAL RESULTS}

The proposed system was tested with flowers bouquet image of size $250 \times 400$. The proposed median filter with variable window size is tested with different noise densities levels:

- very low value of noise densities $3 \%$,

- medium value of noise densities $12 \%$, and

- very large value of noise densities $50 \%$

Three window sizes are taken here, $3 * 3,5 * 5$, and $7 * 7$ window length. These three values of window size are sufficient to analyze the effect of window size on image denoising to remove or at least to reduce the effect of salt and pepper noise.

The salt and pepper noise is inserted with different values of noise density start from 0.03 to 0.12 with step of 0.03 , to represent the noise levels which are low and medium then it jump to $50 \%$ of noise density to represent the high noise level. Median filter with different window size are used for each value of noise density.

The objective evaluation method used here is the evaluation measure for noise that computed as the mean square of the minimum differences. The summary of the results was shown in Table 1. The last row in the Table 1 show noise evaluation measure for 50\% noise density that can be considered as a very high noise level to examine median filter performance with a highly affected noisy image.

The subjective evaluation using human observation can be gained from filtered images with different noise densities. Figure 2 shows that the noise effect decrease with increasing window size but the image lost its blurring. Figure 3 shows the comparison of median filter performance for two values of noise density, which are 0.09 and 0.12 with different window size.

Median filter performance with highly noisy image for different window size is shown in Figure 4. 


\section{CONCLUSION}

Image filtering is one of the most necessary steps in any image processing system and the median filter is the most suitable filter to reduce salt and pepper noise for gray image.

Salt and pepper noise with different noise densities has been inserted with gray image and used in the present work to evaluate median filter performance with different window size. Two different methods has been used in this paper to evaluate quality of noise image: objective quality measure and subjective quality measure. The noise evaluation measure increase with increasing window size that make the observer to think of bad performance of median filter with increasing window size but this not true since the effect of increasing window size appear clearly with high noise density.

The subjective evaluation using human observation can be gained from filtered images with different noise densities that shows the noise effect decrease with increasing window size but the image lost its blurring.

So from these experimental results its concluded that the window sizes of median filter gives a degree of effect on noise removal in images, small window size gives better performance in low noise densities but with high noise density the small window sizes ability to remove noise will be degraded. For large window sizes such $7 * 7$ the noise measure of filtered images is better even in high noise density but will added a degree of blurring effect on the images.

The good performance of median filter with large window size is very clear with high noise density.

Future work we propose a new idea to evaluate the optimized noise filter performance in gray and RGB images compared with standard median filter. High degree of median filter can promise with an acceptable results with high density value of noise.

Table 1. Evaluation of Noise Measure with Respect to different Window Sizes

\begin{tabular}{|c|c|c|c|}
\hline Woise Density & $3 * 3$ & $5 * 5$ & $7 * 7$ \\
\hline $3 \%$ & 6.2729 & 11.5400 & 6.1861 \\
\hline $6 \%$ & 6.7983 & 11.9115 & 16.2457 \\
\hline $9 \%$ & 7.3143 & 12.1405 & 16.4443 \\
\hline $12 \%$ & 8.1045 & 12.4154 & 16.9993 \\
\hline $50 \%$ & 26.8476 & 21.1677 & 23.9797 \\
\hline
\end{tabular}

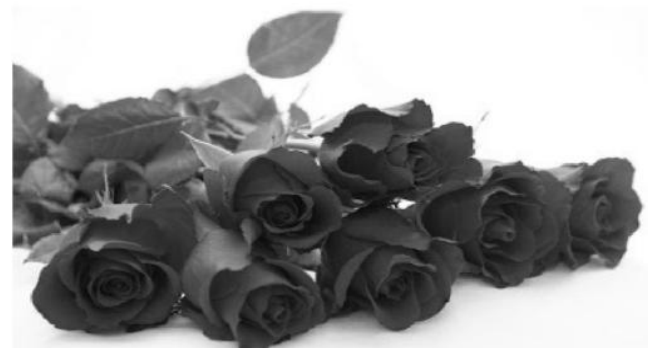

(a)

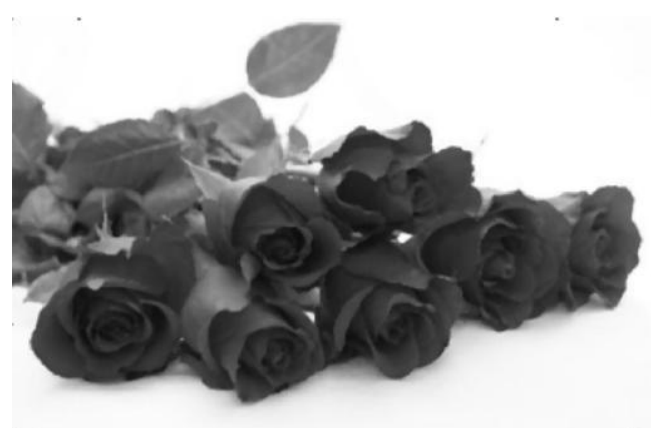

(c)

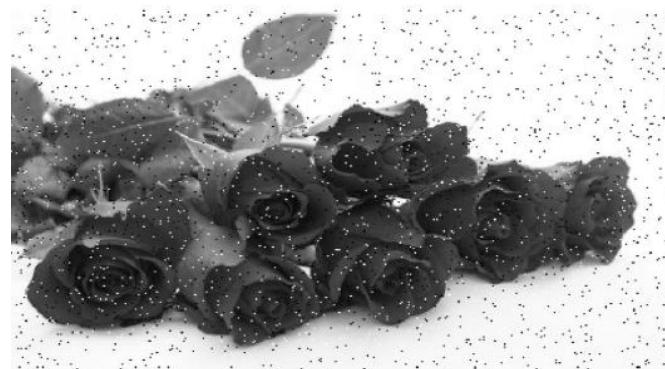

(b)

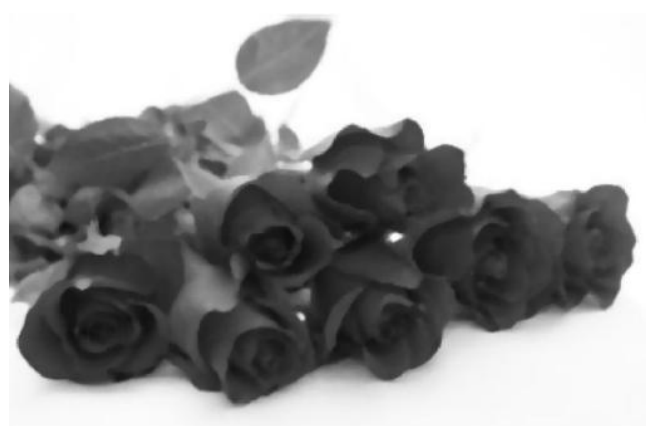

(d) 


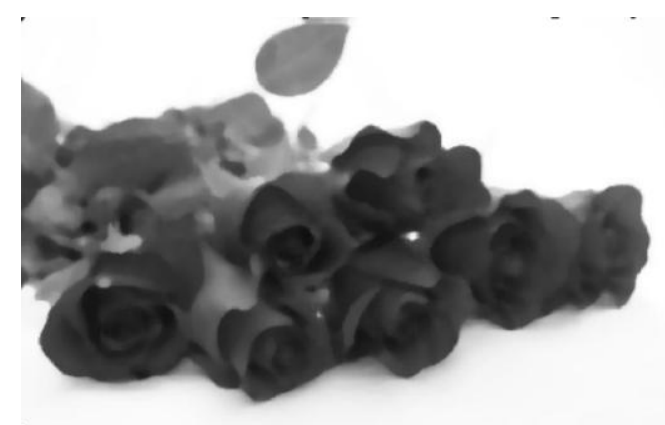

(e)

Fig 2: Performance of median filter of different window size with salt and pepper noise of density $6 \%$. (a). original image, (b). noisy image, (c). filtered image of $3 * 3$ median filter, (d). filtered image of $5 * 5$ median filter, (e). filtered image of $7 * 7$ median filter.

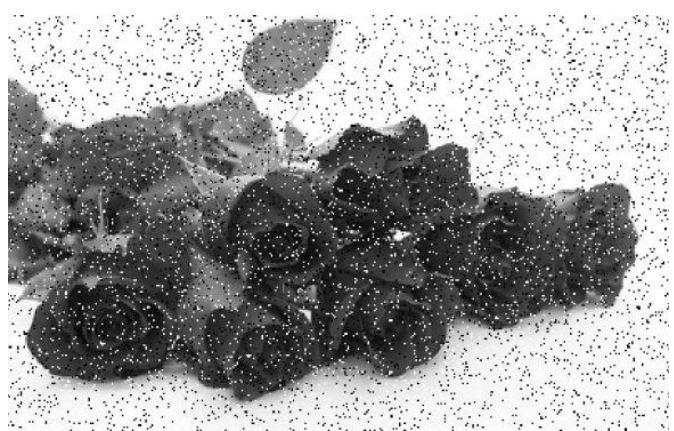

(a)

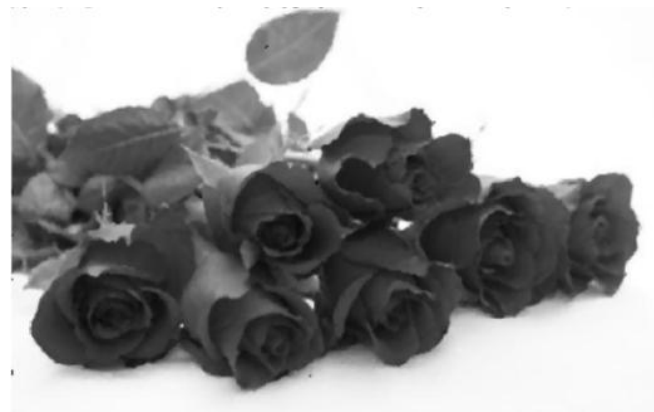

(c)

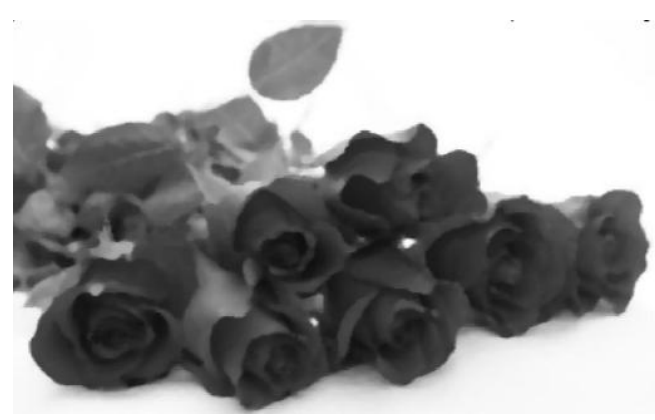

(g)

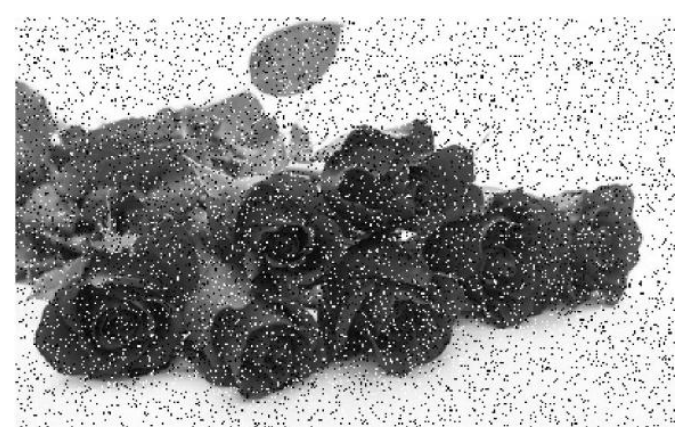

(b)

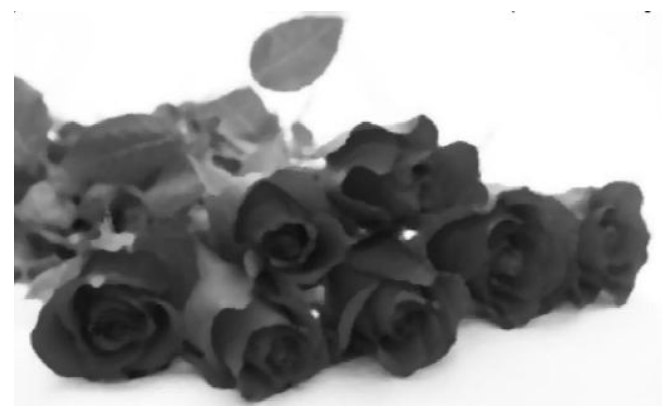

(d)

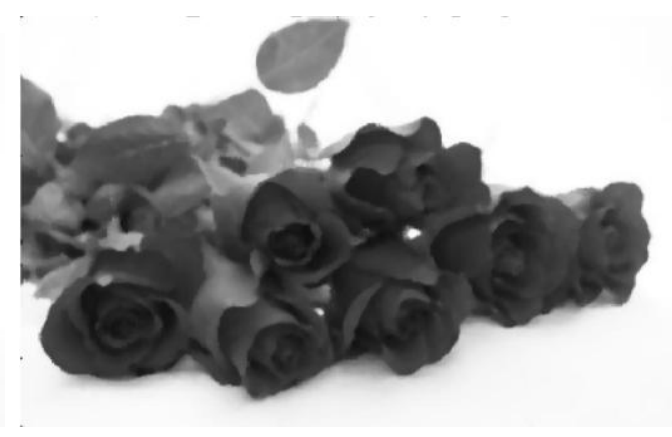

(h) 


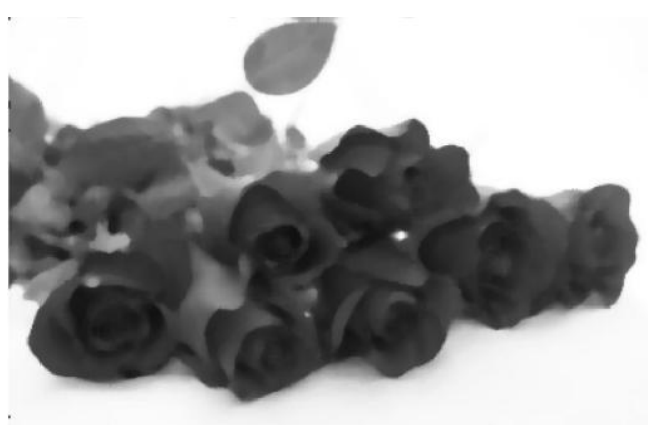

(i)

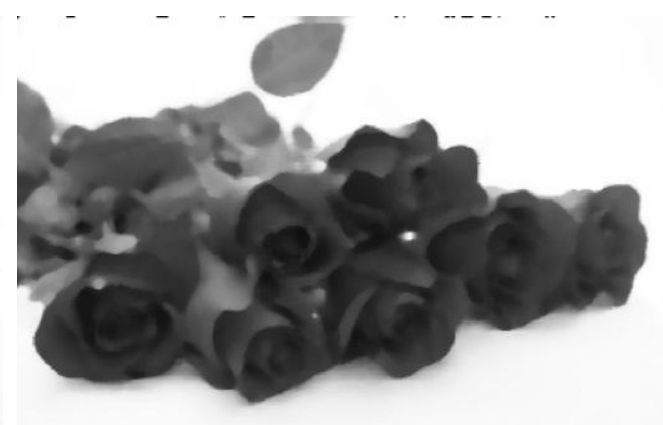

(j)

Fig 3: Performance of median filter of different window size with different noise density. a. noisy image of $9 \%$ noise density, b. noisy image with $12 \%$ noise density, c \& d. filtered image of $3 * 3$ median filter, e \& f. filtered image of $5 * 5$ median filter, $g \& \mathrm{~h}$. filtered image of $7 * 7$ median filter

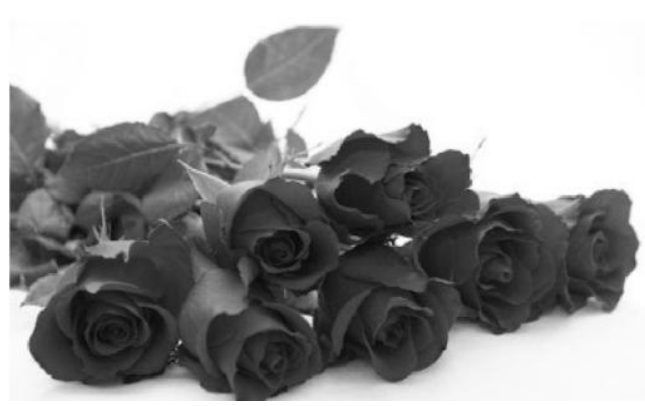

(a)

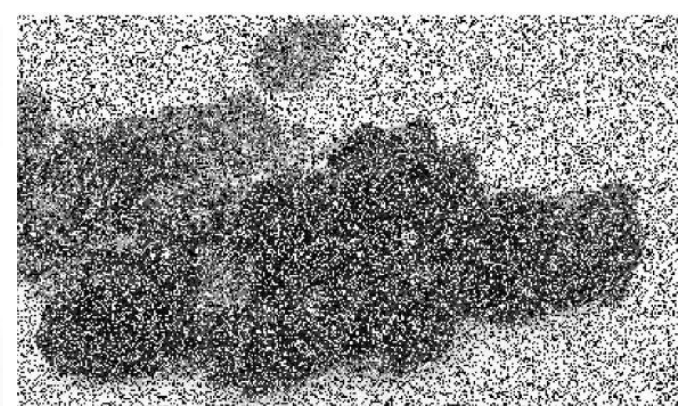

(b)

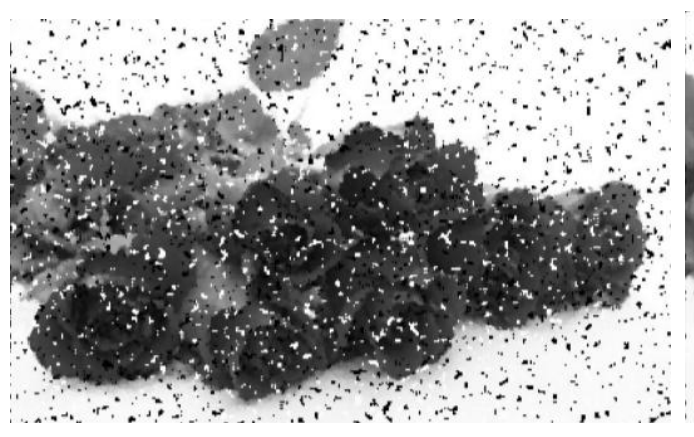

(c)

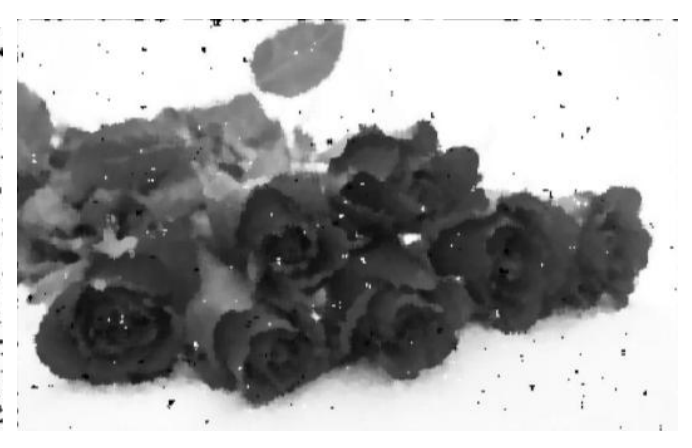

(d)

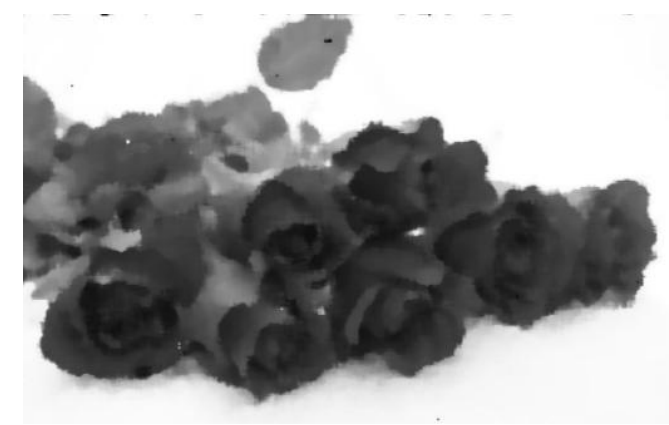

(e)

Fig 4: Performance of $n * n$ median with salt and pepper noise of high noise density of $50 \%$. a. original image, b. noisy image, c. filtered image of $3 * 3$ median filter, d. filtered image of $5 * 5$ median filter, e. filtered image of $7 * 7$ median filter 


\section{REFERENCES}

[1] R. Gonzalez and R.E. Woods, Digital Image Processing. Reading, MA: Prentice Hall,3rd edition, 2007.

[2] N.Rajesh Kumar, J.Uday Kumar,"A Spatial Mean and Median Filter For Noise Removal in Digital Images ",International Journal of Advanced Research in Electrical, Electronics and Instrumentation Engineering, Vol. 4, Issue 1, January 2015.

[3] S. Singh and N. R. Prakash, "Modified Adaptive Median Filter for Salt \& Pepper Noise", International Journal of Advanced Research in Computer and Communication Engineering, vol. 3, no. 1, (2014) January.

[4] M. Karaman, A. Atalar, "Design and Implementation of a General-Purpose Median Filter
Unit in CMOS VLSI", IEEE Journal of Solid-State Circuits, Vol. 25, No. 2, April 1990.

[5] B. Deka and S. Choudhury, "A Multiscale Detection based Adaptive Median Filter for the Removal of Salt and Pepper Noise from Highly Corrupted Images", International Journal of Signal Processing, Image Processing and Pattern Recognition, vol. 6, no. 2, (2013) April.

[6] M. S. Nair, K. Revath and R. Tatavarti, "Removal of Salt-and Pepper Noise in Images: A New Decision-Based Algorithm", Proceedings of the International Multi Conference of Engineers and Computer Scientists, vol 1, (2008), Hong Kong.

[7] Eskicioglu AM, Fisher PS. Image quality measures and their performance. IEEE Trans Commun. 1995;43:2959-65. 